\title{
TO PROTECT USER'S PASSWORD FROM THEFT BY USING RFID AND MEMS TECHNOLOGY
}

\author{
Aghade Akshata ${ }^{1}$,Bhandge Neha $\mathrm{A}^{\mathbf{2}}$, Kawade Supriya $\mathrm{E}^{\mathbf{3}}$, Thawal Seema $\mathrm{M}^{4}{ }^{4}$ \\ ${ }^{1}$ Student, Computer Engineering, SND college of Engineering Yeola, SPPU, Maharashtra, India \\ akshataaghade94@gmail.com \\ ${ }^{2}$ Student, Computer Engineering, SND college of Engineering Yeola, SPPU, Maharashtra, India \\ nehabhandge18@gmail.com \\ ${ }^{3}$ Student, Computer Engineering, SND college of Engineering Yeola, SPPU, Maharashtra, India \\ priyakawade12@gmail.com \\ ${ }^{4}$ Student, Computer Engineering, SND college of Engineering Yeola, SPPU, Maharashtra, India \\ seemathawal93@gmail.com
}

Guided By: Prof. Shaikh I.R. Assistant Professor of Computer Engineering, SND college of Engineering Yeola, SPPU, Maharashtra, India

\begin{abstract}
In this paper, MEMS (Micro Electro Mechanical System) is implemented using Embedded controller PIC 16F877A. MEMS can be used to sense motion. Microcontroller detect any motion while they are occurs or not. The user can assign a password by fixed set of motions as decided by them. In that there are three techniques uses for user's authentication. Firstly Microcontroller senses any motion which is matches with exist motion password, then first phase will unlock and it goes into next phase is human interface method of keypad that is traditional where the user enters the predefined password, if it is matched with the predefined password then second phase will be unlock. Then user will directly enter into the final phase which is a RFID card that is read by a RFID reader module connected to the Micro controller if the card is matched the final phase get completed and the desired operation will start. A buzzer is used for an indication of wrong password. This buzzer is ON until right password matches with existing password.
\end{abstract}

Key Words: -Codebooks, Key Logger, PIC Microcontroller, Secret Little Functions, RFID, MEMS.

\section{INTRODUCTION}

Now a days the Online Banking has entered into our daily life. We have also become usually to harmful work, such as paying money using credit cards. While we enjoy it's easy to computing. Many websites ask user for their User ID and passwords for authentication. Once a user's enters its ID and the corresponding password are protecting by theft, \& the theft can do anything with user's account, which can lead to harmful for the user. By observing all this it becomes a very critical job to protect the user ID and Password from theft. The secure socket layer protocol [1] for transferring secret data over the web is well-known in recent research, but mostly used websites still use a weak protection for user authentications through a plaintext password and user ID. Meanwhile, even though a password can be transmit through a protected channel.

This authentication approach is still support the following attacks:

[1]. Phishing attacks:-In phishing attack the attacker try to fetch the personal information of user like the credit card details user name and password [2].

[2]. Password Stealing Trojan:- Contain or install harmful and unauthorized codes. Examples include: a) key loggers capturing keystrokes in the machine; and b)
Trojan Redirectors redirecting end-users network traffic to a desired location [3].

[3]. Shoulder Surfing:- Steals others private information by looking over victim's shoulders [4] or capturing victim pictures and videos using cameras. There are many different ways to prevent victims from some specified attacks. However, we cannot prevent all the attacks at the same time.

\section{LITERATURE SURVEY}

Now-a-days, ATM Machines, Bank lockers is used. There are huge chances for the hackers to theft the User ID and password or user's credit card.

In they have use the PIC16F877A microcontroller for controlling all input and output devices [5].

In Secret Little Function- It is the user define or userspecified function or program [6]. This function is only known between the server and the user, they are very secure, simple functions [7].

In MEMS (Micro Electro Mechanical System) controlling of password theft by using LIS3LVO2DQ specified MEMS has been used in advance security[6]. 
In RFID READER It is a fast affordable and automatic identification technology that uses radio frequency to transfer data between RFID Reader and RFID Tag [7]. RFID circuit is a single solid state memory chip.

In Codebook- This is need to protecting the whole system in case some user loses their codebook to theft [8]. Our system has sufficient computing power to run a cryptographically secure RNG (Random Number Generator)[8]. In that the system will not be compromised and the user can easily ask for a new codebook without changing the parameters of the RNG [6].

\section{PROPOSED SYSTEM}

We have been proposed the system which is robust in nature. We have strong security mechanism. In that there are three phases of locks each and every phase are depends on previous phase. In that firstly enter the password it will check that it is correct or not then system executes the second module which is depends on previous one.

\subsection{There are three phases in our system like:}

\section{- MEMS:}

Which is one kind of sensor that are used to sense the human motion it capture all the analog signals or real time or real world signal. It is also used to capture the motion in the form of biometric.

\section{- Features:}

1. $2.16 \mathrm{v}$ to $3.6 \mathrm{v}$ single supply operation.

2. Embedded self test.

3. Eco-pack complaint.

\section{- RFID:}

Which is small ATM card, Credit card like printed card this card store our personal information which is helpful for system to guess our real password or plan text password.

\section{- RFID Modules:-}

RFID system consist of three components as

1) RFID Tag(Transponder)

2) Tag Reader(Interrogator)

3) Computer or Processor.

\subsection{Secret Little Function:}

In that user use the tradition method of authentication i.e. keyboard means user enter the PIN to unlock the system. In that this special function are used for strong security.

\subsection{Microcontroller :}

In that system microcontroller plays the vital important role to controlling all the hardware parts of our system. It provide the interconnection between input and output part. In that they also convert the analog signal into digital signal part or signal also provide the synchronization between that signals.

\subsection{Features:-}

[1].High performance RISC CPU.

[2].Operating speed:200ns, $20 \mathrm{MHz}$ instruction cycle.

[3].Operating voltage: $4.0-5.5 \mathrm{~V}$

Microcontroller has five component to manage and control the all input and output devices that is used in system.

\section{1] ADC:}

ADC is a device that converts physical quantity to a digital number that represent quantity amplitude. Analog to Digital Converter that's converts the real time analog signal into digital form. This signal controls all the real time motion which is captured by MEMS.

\section{2] I/O:-}

$\mathrm{I} / \mathrm{O}$ is input and output. Input is password through keypad and output is display screen.

\section{3] Pwm:-}

PWM is abbreviated as Pulse Width Modulation. a modulation technique used to encode a message into a pulsing signal. PWM is a technique for getting analog results with digital means. Digital control is used to create a square wave, signal switched between on and off.

\section{4] Uart:-}

UART is abbreviated as Universal Asynchronous Receiver/Transmitter .In asynchronous transmitting , teletype -style UARTs send a "start" bit, five to eight data bits ,least-significant first, an optional "parity" bit and then one ,one and a half ,or two "stop" bits.

\section{5] Power Supply:-}

A power supply is a electronic device which is used to give an electric energy supply. It is also called as electric power converter. The large number of voltage is converted into $5 \mathrm{~V}$.

\subsection{Output}

\section{- Dc Motor:-}

A motor driver is a designed to drive an electromagnetic load, such as a brushed or brushless motor, stepper motor or relay.

\section{- Display:-}

It is output part. It is either LCD or LED device. It can show or view the user's entered password. Which is enters in traditional keyboard method. This display screen is also shows the messages that messages helpful to user for operating system. 


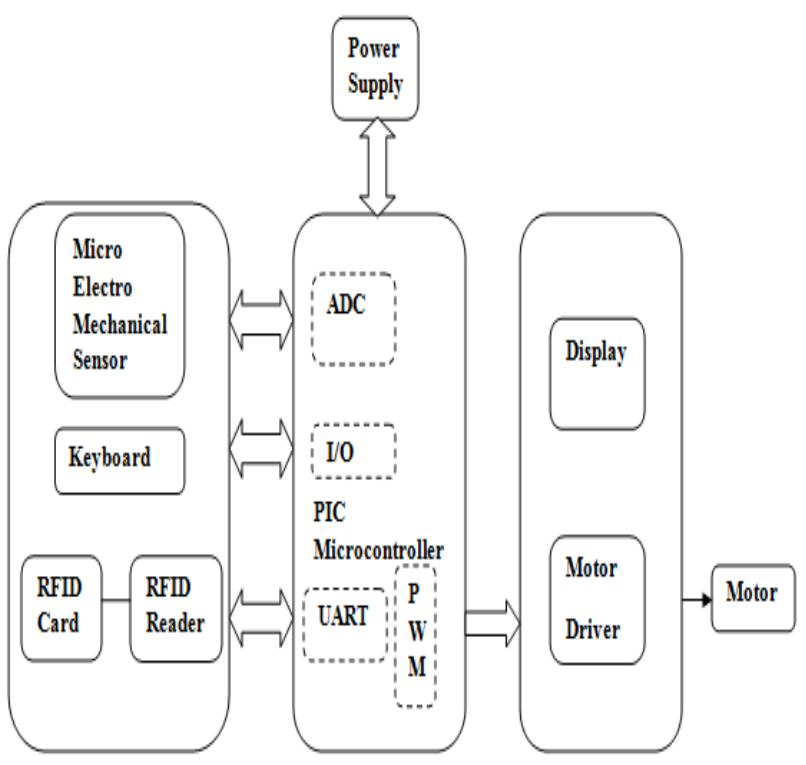

Fig 1. Block diagram of proposed system

\section{CONCLUSION}

Hence, we have proposed the system to protect user's password from theft by using RFID and MEMS Technology. This system is used to select a virtual password scheme ranging from weak security to strong security. We further proposed several functions serving as system confirm functions and provided a security analysis. We analyse how the proposed schemes defend instead of phishing, key-logger, shoulder-surfing attacks, and multiple attacks. It is suggested to utilize that important account like bank accounts. How to prevent users' passwords from being stole by adversaries.

\section{ACKNOWLEDGEMENT}

I wish to express my sincere thanks and deep sense of gratitude to respected guide Prof. Shaikh I.R. in Department of Computer Engineering of SND college of Engineering \& Research Center Babhulgaon Yeola 423401,for the technical advice, encouragement and support us \& guide for the guidance about project related queries.

\section{REFERENCES}

[1]. T. Dierks and C. Allen, The TLS Protocol-Version 1.0, IETF RFC 2246,Jan. 1999.

[2]. Anti-Phishing Working Group. [Online]. Available: http://www.antiphishing.org

[3]. [Online].Available:http://en.wikipedia.org/wiki/Key-1 ogger

[4]. [4] V. A. Brennen. (2004). Cryptography Dictionary, vol.

2005,1.0.0ed.[Online].Available:http://cryptnet.net/fdp /crypto/crypto dict/en/cryptodict.html

[5]. J. R. Fisher \& Carla Beaudet, The Functional
Description of PIC16F877A Functions, April 26, 2005

[6]. C. T. Leondes, Mems/Nems Handbook techniques and applications, Volume 4: Sensorsand actuators (Springer, USA, 2006)

[7]. J. Zheng, J. Li, M. J. Lee, and M. Anshel, "A lightweight encryption and authentication scheme for wireless sensor networks," Int. J.Security Netw., vol. 1, nos. 3-4, pp. 138-146, 2006.. Herzberg and A. Gbara, "Trustbar: Protecting (even naive) web users from spoofing and phishing attacks, "Cryptology ePrintArchive,Report2004/155, 2004. http://eprint.iacr.org/2004/155.

\section{BIOGRAPHIES}

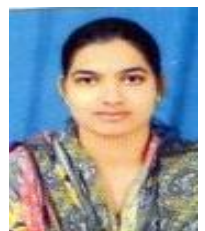

Akshata B. Aghade

She is student of computer Engg. At SND COE \& RC,Yeola Under university of Pune. akshataaghade94@gmail.com

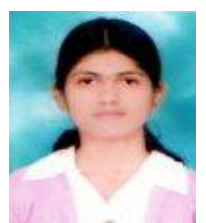

Neha A. Bhandge

She is student of computer Engg. At SND COE \& RC,Yeola Under university of Pune.nehabhandge18@gmail.com

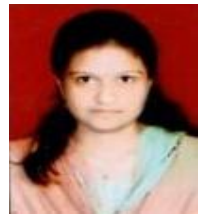

Supriya E. Kawade

She is student of computer Engg. At SND COE \& RC,Yeola Under university of Pune.priyakawade12@gmail.com

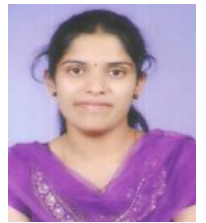

Seema M. Thawal

She is student of computer Engg. At SND $\mathrm{COE} \& \mathrm{RC}$,Yeola Under university of Pune.seemathawal93@gmail.com 